\title{
Application Progress of Modified Early Warning Score in General Departments
}

\author{
Rong Tang, Fanxin Xuan, Xiaowei Qin, Lanping Shi* \\ Department of Medicine, Yangtze University, jingzhou, China \\ Email: 1129934527@qq.com, *shilp@hku-szh.org
}

How to cite this paper: Tang, R., Xuan, F.X., Qin, X.W. and Shi, L.P. (2019) Application Progress of Modified Early Warning Score in General Departments. Yangtze Medicine, 3, 225-234.

https://doi.org/10.4236/ym.2019.33022

Received: April 18, 2019

Accepted: June 25, 2019

Published: June 28, 2019

Copyright $\odot 2019$ by author(s) and Scientific Research Publishing Inc. This work is licensed under the Creative Commons Attribution International License (CC BY 4.0).

http://creativecommons.org/licenses/by/4.0/ (c) (i) Open Access

\begin{abstract}
Modified Early Warning Score system can help medical staff to identify patients with "potential critical illness" at an early stage and effectively reduce the death rate. In 2001 the NHS recommended MEWS for general practice assessment. However, there are few studies on MEWS in general departments, and the specific application effect is still being explored. Therefore, this paper summarizes the application advantages, application methods and application effects of MEWS in general departments at home and abroad, so as to provide some theoretical basis for the development of MEWS in general departments. After summary, it was found that although MEWS evaluation indicators are simple and easy to obtain, and can facilitate the communication of medical conditions, MEWS has a poor ability to identify "potential critically ill patients" in general departments. The role of predicting the prognosis of patients in general departments remains to be explored. A large number of multi-center, large-sample and large-range studies are needed to provide a basis for the application of MEWS in general departments.
\end{abstract}

\section{Keywords}

Modified Early Warning Score, General Departments, Potential Critical Illness Patients

\section{Introduction}

"Potential critical illness" refers to patients who do not have any obvious manifestation of organ failure on the surface, but without timely and effective intervention and treatment, their condition may rapidly deteriorate within a few hours or days, or even lose their lives [1]. Modified Early Warning Score (MEWS) was developed and improved by Subbe CP on the basis of EWS [2] [3]. Studies have shown that MEWS can effectively help medical staff to identify pa- 
tients with "potential critical illness" in an early, accurate and objective manner. It not only promotes the early implementation of relevant medical measures, but also reduces the death rate [4] (see Table 1). In 2001, the National Health Service (NHS) recommended it as one of the methods for the assessment and communication of medical staff in medical institutions, and recommended MEWS in the emergency department, Intensive Care Unit (ICU) and general departments. However, compared with the research on MEWS in emergency department and ICU, the application of MEWS in general departments lacks big data support, and the application effect is still being explored [5]. Therefore, the application of MEWS in general departments at home and abroad was reviewed, so as to provide a basis for the development of MEWS in general departments.

\section{Application Background and Potential Analysis of MEWS in General Departments}

\subsection{Application Background of MEWS in General Departments}

The application of MEWS in emergency department and ICU provides experience for general departments to develop MEWS [6]. Some studies have pointed out that among 254 emergency departments in UK, 87\% of hospitals use MEWS to evaluate and predict patients' conditions, and $93 \%$ of hospitals give recognition and support to this tool, and point out that MEWS can effectively predict whether emergency patients need to be admitted to general departments or transferred to ICU [7]. For ICU patients, effective condition assessment is the first step to save patients. The most authoritative and most widely used assessment scale is the Acute Physiology and Chronic Health Evaluation (APACHE II), which can predict the severity of patients with $90 \%$ accuracy. Some studies have pointed out that MEWS can also reach the same level, both of which have good clinical resolution [8]. Therefore, it is of practical significance to promote the application of MEWS in general departments based on its experience in emergency department and intensive care unit.

\subsection{Advantage Analysis of MEWS Application in General Departments}

\subsubsection{MEWS Evaluation Indicators Are Easy to Be Collected and Calculated} MEWS includes body temperature, heart rate, respiration, systolic blood pressure and consciousness. The consciousness with A (Alert), V (Reacting to voice),

Table 1. Modified Early Warning Score (MEWS).

\begin{tabular}{cccccccc}
\hline Score & 3 & 2 & 1 & 0 & 1 & 2 & 3 \\
\hline Heart rate/min & & $\leq 40$ & $41-50$ & $51-100$ & $101-110$ & $111-129$ & $\geq 130$ \\
Systolic blood pressure & $\leq 70$ & $71-80$ & $81-100$ & $101-199$ & & $\geq 200$ & \\
consciousness & & & & $\mathrm{A}$ & $\mathrm{V}$ & $\mathrm{P}$ & $\mathrm{U}$ \\
breathing $/ \mathrm{min}$ & & $<9$ & & $9-14$ & $15-20$ & $21-29$ & $\geq 30$ \\
temperature $/{ }^{\circ} \mathrm{C}$ & & $\leq 35.0$ & $35.1-36$ & $36.1-38$ & $38.1-38.5$ & $\geq 38.6$ & \\
\hline
\end{tabular}


P (Reacting to pain), U (Unresponsive) score [9]. The other four indexes set several parameter intervals, and each parameter interval corresponds to a different score. During the evaluation, the corresponding parameter interval and score were determined according to the actual value of each index of the patient. Finally, the score values of the 5 items were added to obtain the total score. The higher the score is, the worse the patient's current condition is. The score 0 - 4 is classified as mild disease, 5 - 8 as severe disease, and 9 as critical disease [4]. The vital signs of patients in general departments are the data that nurses must collect every day. MEWS can reflect the severity of this patient's condition instantly by simple calculation, which is feasible without additional manpower and material resources. Dalton's study also points out that nurses in general departments are willing to evaluate patients' MEWS on time and report abnormal conditions to nurse practitioners or doctors, which indicates that the implementation of MEWS in general departments is feasible [10] [11] [12].

\subsubsection{Increase Effective Communication among Medical Staff}

Patients with "potential critical illness" often show abnormal physiological indicators, such as: change in consciousness, abnormal blood pressure, shortness of breath, arrhythmia, decreased oxygen saturation $\left(\mathrm{SpO}_{2}\right)$, or decreased urine volume, in a few hours or days before the condition changes, which are also known as "warning information" [11] [12]. However, in the medical work, it is difficult for nurses to judge whether the positive signs of patients indicate serious changes from the overall perspective. There is often a delay in reporting a patient's positive signs to a doctor or a failure to do so. Secondly, nurses tend to judge patients' conditions based on clinical experience and intuition, resulting in "wrong judgment" or "omission" of patients' conditions [10], and then miss the best treatment time, leading to irreversible consequences. Combined with vital signs and consciousness, MEWS covers the respiratory, circulatory and nervous systems, and reflects the overall condition of the patient with the most simple and direct score. MEWS can help the other party to grasp the patient's condition faster and more effectively [13].

\section{Application of MEWS in General Departments}

\subsection{Specific Departments Applicable to MEWS}

In 2001, the National Health Service (NHS) recommended medical institutions and medical staff to use MEWS for disease assessment and communication [4], and recommended the application of MEWS in emergency department, intensive care unit and general department [14] [15] [16]. In the report of Chubu Tokushukai hospital in Okinawa, Japan, MEWS was applied to all the general departments of the hospital. Clinical nurses evaluated MEWS for patients every day. When the total score reached 7 , nurses and doctors conducted relevant treatment. The results confirmed that MEWS can effectively reduce the rate of cardiac arrest in inpatients [17]. There are few general departments applying MEWS in China, and most of them focus on the studies of single department 
[18] [19] or single disease [20] [21], such as the use of MEWS in cardiology, and the application value of MEWS in cardiac arrest patients, etc. At present, the specific departments applicable to MEWS are still not clear. The literature was summarized in Table 2.

\subsection{Dynamic MEWS Can Better Reflect Patients' Conditions}

Dynamic MEWS score can better reflect patients' conditions in real time [8]. Nishjima once evaluated the MEWS once a day for all patients in the general department, the corresponding physiological indicators recorded into the nursing system, by the system automatically calculate the MEWS score, when reached 7 points, the system automatically alarm, ward nurses need to report the attending doctor, for the treatment of patients, during which continue to evaluate three times a day, if discover the patients need a higher level of medical care facilities, are immediately turned to ICU, unless the patient give up treatment agreement (Do Not Resuscitate, DNR) [17]. Most domestic studies have studied the degree of prediction of disease changes in patients with a certain static MEWS score, with the most related studies on single score at admission [22]. Su Yanhong evaluated 200 patients in the department of cardiothoracic surgery once a shift when they were admitted to the hospital and did not use ECG monitoring after surgery. When abnormal vital signs were found, it was necessary to report to the doctor at any time until the single score $<2$ or the total score $<4$, but the treatment process was not described in detail [23].

\subsection{Specific Application Process of MEWS}

Penglingli applied MEWS in the general department of the hospital in 2014, and formed a relatively perfect application process: MEWS should be evaluated when patients are admitted to the hospital, returned to the ward after surgery, returned to the ward after surgery for $6 \mathrm{~h}$, and transferred to the hospital. If single item $>2$ or total score $\geq 4$, the nurse should evaluate once per shift, and report the patient's abnormal vital signs and the most urgent problems to the doctor in time until the score is reduced to normal. When the total score of MEWS is greater than 5, the nurse should increase the number of monitoring, follow the doctor's advice for ECG monitoring if necessary, inform the superior doctor and [1] remind the "seriously ill" doctor's advice; When the total score of MEWS is greater than 6, in addition to the above three points, it is also necessary to prepare for rescue, including opening the vein access, preparing rescue materials, and helping doctors contact ICU nurses [1].

\section{Limitations of MEWS Application in General Departments MEWS Combined with Specialist Indicators Is More Accurate}

The advantage of MEWS for general departments is that it is easy to evaluate projects. However, due to the changeability of patients' conditions, the variety of diseases and the complexity between different departments, MEWS lacks accuracy 
Table 2. Domestic and foreign studies on MEWS in single department or single disease.

\begin{tabular}{|c|c|c|c|c|c|}
\hline No. & Year & Author & Title & Journal & Page \\
\hline & 1999 & $\begin{array}{l}\text { McGloin, H } \\
\text { Adam, S K } \\
\text { Singer, M }\end{array}$ & $\begin{array}{c}\text { Unexpected deaths and referrals } \\
\text { to intensive care of patients on } \\
\text { general wards. Are some cases } \\
\text { potentially avoidable? }\end{array}$ & ORIGINAL PAPERS & 255 \\
\hline
\end{tabular}

Conclusion

2005
Palients with obvious clinical indicators of acule deterioration can be overlooked or poorly managed on the ward. This may lead to potentially avoidable unexpected deaths or to a poorer eventual outcome following ICU admission. Early recognition and correction of abnormalities may result in outcome benefit, but this requires further invesligation.

Subbe, C P

Williams, E

Fligelstone, L

Gemmell, L
Does earlier detection of critically ill patients on surgical wards lead to better outcomes?
Ann R CollSurg Engl

$226-232$

There is evidence that simple algorithms based on bedside observations can identify a large proportion of sick patients on general wards. Non-randomised studies have shown mixed results on impact of these interventions on mortality, cardiopulmonary arrests and intensive care admissions. The majority of studies do not specifically address surgical patients. Award-based randomised trial from the UK seems to suggest improved mortality following the introduction of a Critical CareOutreach service with an Early Warning Score.

$$
\begin{array}{cc}
\text { Ying-xian Li } & \text { Study on the prognostic value } \\
\text { Hong-mei Ye } & \text { of improved early warning score in } \\
\text { Min-li Tang } & \text { patients with severe pancreatitis }
\end{array}
$$

Rational

clinical medication
MEWS and APACHE scoring have the same predictive value for the prognosis of SAP patients, but the MEWS scoring system is simple in structure, convenient in data acquisition, time-saving and cost-saving, and can be used to predict the prognosis of SAP patients.

$\begin{array}{cc}\text { Reini, Kirsi } & \text { The prognostic value of the } \\ \text { Fredrikson, Mats } & \text { Modified Early Warning Score } \\ \text { Oscarsson, Anna } & \text { in critically ill patients }\end{array}$

European Journal

of Anaesthesiology
This study shows that the Modified Early Warning

Score is a useful predictor of mortality in the ICU, 30-day mortality and length of stay in the ICU

Smith, Tim
Hartog, Dennis
Moerman, Tineke
Accuracy of an expanded early warning score for patients in general and trauma surgery wards

An EWS of 3 or more is an independent predictor of major adverse events in patients admitted to a general and trauma surgery ward.

$\begin{array}{cc}\text { Cooksley, T } & \text { Effectiveness of Modified Early } \\ \text { Kitlowski, E } & \text { Warning Score in predicting } \\ \text { Haji-Michael, P } & \text { outcomes in oncology patients }\end{array}$

The currently used track and trigger systems have poor discriminatory value in identifying Oncological patients at risk of deterioration. An adapted score more focused upon the key predictive physiological parameters in this population needs to be developed to produce a more effective tool.

Jung-Wan Yoo, JuRy Lee, Youn
Kyung Jung
A combination of early
warning score and lactate to predict intensive care unit ORIGINAL ARTICLE transfer of inpatients with severe sepsis/septic shock 


\section{Continued}

2014

8

Conclusion

2015

Conclusion

2016

10

Conclusion

2016

11

Conclusion

2016

12

Conclusion
Application of early warning score in

identifying "potential critical illness" in surgical wards
Nursing practice and research

$58-59$

EWS can identify patients with "potential critical illness" in the surgical ward. The earlier it is identified, the more opportunities for early medical intervention and the higher survival rate of patients.EWS can also determine the prognosis of critically ill patients, especially in elderly patients with significant correlation.

$$
\begin{gathered}
\text { Li-li Wang } \\
\text { Jie-fu Du } \\
\text { Wei Chen } \\
\text { Li-jingJia } \\
\text { Hai-yan Zhu }
\end{gathered}
$$

Methodological research

$1680-1684$

elderly emergency patients

NEWS can reflect the actual situation of respiration and heart rate of elderly emergency patients more accurately and effectively. Compared with MEWS score, NEWS is more suitable for assessing the outcome and prognosis of elderly emergency patients.

$$
\text { Li Yang }
$$

Yong-mei Zhang

Fei Chen

Mei-lin Lu

Ting-mei Zhou

Ai Liu

\section{Application of modified early}

warning score in disease risk management of elderly inpatients in digestive surgery department
Nursing research

$1633-1635$

Early use of MEWS score to provide early warning for hospitalized elderly patients with digestive surgery is conducive to medical staff's early detection and improvement of their conditions Timely treatment measures, effectively reduce the risk of deterioration.

Yan-hong Su

Lun-fang Huang

$\mathrm{Lu}$-si Yu
Application analysis of the improved early warning scoring system in the nursing of "potential critical patients" in cardiothoracic surgery
Contemporary nurse

57-59

For patients with cardiothoracic surgery, the improved early warning scoring system is adopted for disease observation, which is conducive to the evaluation of the condition change of "potential critical patients" in cardiothoracic surgery, and can timely detect the condition change of patients, give corresponding nursing measures, and carry out intervention, effectively avoiding the disease of patients. In order to reduce the incidence of accidents and the rate of re-referral to the ICU of cardiothoracic surgery, and improve the satisfaction rate of patient satisfaction survey.

$$
\begin{gathered}
\text { Isao Nishijima } \\
\text { Shouhei Oyadomari }
\end{gathered}
$$

\author{
Use of a modified early warning \\ score system to reduce the rate \\ of in-hospital cardiac arrest
}

Journal of intensive Care $1-6$ decreased owing to the introduction of the system, as the system enables early interventions for patients who have the possibility of acute deterioration.

and sensitivity in the evaluation of patients' conditions [13] [24] Delgado has changed the consciousness score from AVPU to GCS score on the basis of MEWS, and the results confirmed that the improved version of MEWS has good reliability and validity [25]. Ye explore BISAP, MEWS, $\mathrm{Ca}^{+}$, red blood cell width as Acute Pancreatitis, Acute Pancreatitis, AP) the influence of the factors, found that when used MEWS and other indicators that can effectively evaluate the occurrence of AP, but separate MEWS failed to predict the occurrence of AP [26]. Skaleaky M also established pediatric early warning score on the basis of EWS 
with specialized indicators, and determined that when the optimal cut-off point was 2.5, the maximum AUC of the score was 0.81, suggesting that it could help pediatric nurses to identify children with deteriorating conditions in the early stage [27], indicating that MEWS combined with specialized indicators was more effective in general departments.

\section{Effects of MEWS in General Departments}

\subsection{The Ability of MEWS to Predict the Prognosis of Patients in General Departments Remains to Be Explored}

The ability of MEWS to determine the prognosis of patients in general departments remains to be explored [28] [29]. Tirotta collected demographic data of 31 patients with continuous sepsis infection in the medical ward, the rate of transfer to the ICU, and MEWS, and found that MEWS had little risk value in predicting the in-hospital death of sepsis patients in the medical ward [30]. More systematic evaluation directly points out that: MEWS is not good at identifying and predicting the prognosis of patients with "potential critical illness" in general departments [31] [32]. However, some studies suggest that MEWS is suitable for general departments. Lu xiaomin has analyzed the EWS score of 88 patients who were unplanned transferred to ICU in the surgical ward within 24 hours before the transfer, and found that EWS can identify patients with "potential critical illness" and help to determine the prognosis of patients [33]. Wenting Huang compared the NEWS, the BAP-65 scores, Chronic Respiratiory Early Score (CREW) three Score to predict the prognosis of patients with acute exacerbation of Chronic obstructive pulmonary disease period. It was found that the AUC of death predicted by NEWS was 0.878 , and that of ICU admission was 0.826 , indicating high performance [34].

\subsection{Poor Ability of MEWS in Identifying Patients with "Potential Critical Illness" in General Departments}

Some studies have pointed out that although MEWS is recommended as an evaluation tool for many departments, it is not based on a large number of clinical studies, so its effectiveness cannot be guaranteed [5], which is consistent with the views of many researchers in systematic evaluation [16] [35] [36]. Cooksley has retrospectively collected the MEWS scores of 840 patients in the oncology department to explore its ability to predict ICU transfer and death within 30 days, and found that MEWS has poor ability to identify cancer patients at risk of deterioration [9]. Therefore, in order to effectively promote MEWS at home and abroad, a large number of multi-center, large-sample and large-range clinical studies need to be improved [28].

\section{Summary}

Patients with "potential critical illness" have high risk of irreversible negative events and poor prognosis. Early identification of patients with "potential critical 
illness" and early implementation of relevant intervention and nursing can effectively reduce the death rate and improve the prognosis of patients [2] [3]. The main reason is that MEWS evaluation indicators are easy to collect and calculate. The simple score can reflect the overall condition of patients and facilitate communication between medical staff. However, due to the lack of unified application mode and application timing of MEWS, there are different opinions about the application effect of MEWS in general departments at home and abroad. According to the authors, the application of MEWS in general departments needs a unified application process, and only by adding specialist indicators and increasing the ability of MEWS to identify patients with "potential critical illness" can it be implemented in general departments.

\section{Conflicts of Interest}

The authors declare no conflicts of interest regarding the publication of this paper.

\section{References}

[1] Peng, L., Li, Y., He, A., et al. (2014) Establish Disease Early Warning Workflow to Ensure Patient Safety. China Nursing Management, No. 12, 1250-1254.

[2] Franklin, C. and Mathew, J. (1994) Developing Strategies to Prevent Inhospital Cardiac Arrest: Analyzing Responses of Physicians and Nurses in the Hours before the Event. Critical Care Medicine, 22, 244-247. https://doi.org/10.1097/00003246-199402000-00014

[3] Mcgloin, H., Adam, S.K. and Singer, M. (1999) Unexpected Deaths and Referrals to Intensive Care of Patients on General Wards. Are Some Cases Potentially Avoidable? Journal of the Royal College of Physicians of London, 33, 255.

[4] Chen, Z. (2014) Calibration of Improved Early Warning Score for Clinical Use in Surgical High-Dependence Wards. Tianjin Medical University, Tianjin.

[5] McGaughey, J., et al. (2007) Outreach and Early Warning Systems (EWS) for the Prevention of Intensive Care Admission and Death of Critically Ill Adult Patients on General Hospital Wards. Cochrane Database of Systematic Reviews, No. 3, CD005529. http://www.thecochranelibrary.com

[6] Sun, P. and Li, F. (2016) Clinical Application of Improved Early Warning Score in Emergency Patients at Home and Abroad. Journal of Nursing, No. 3, 36-39.

[7] Griffiths, J.R. and Kidney, E.M. (2011) Current Use of Early Warning Scores in UK Emergency Departments. Emergency Medicine Journal, 29, 65-66. https://doi.org/10.1136/emermed-2011-200508

[8] Meng, X. (2008) Critical Score-Evaluation, Prediction and Treatment. People's Health Publishing House, Beijing.

[9] Cooksley, T., Kitlowski, E. and Haji-Michael, P. (2012) Effectiveness of Modified Early Warning Score in Predicting Outcomes in Oncology Patients. QJM, 105, 1083-1088. https://doi.org/10.1093/qjmed/hcs138

[10] Dalton, M., Harrison, J., Malin, A., et al. (2018) Factors That Influence Nurses' Assessment of Patient Acuity and Response to Acute Deterioration. British Journal of Nursing, 27, 212-218. https://doi.org/10.12968/bjon.2018.27.4.212

[11] Roney, J.K., Whitley, B.E., Maples, J.C., et al. (2015) Modified Early Warning Scor- 
ing (MEWS): Evaluating the Evidence for Tool Inclusion of Sepsis Screening Criteria and Impact on Mortality and Failure to Rescue. Journal of Clinical Nursing, 24, 3343-3354. https://doi.org/10.1111/jocn.12952

[12] Wang, A., Fang, C., Chen, S., et al. (2016) Periarrest Modified Early Warning Score (MEWS) Predicts the Outcome of In-Hospital Cardiac Arrest. Journal of the Formosan Medical Association, 115, 76-82. https://doi.org/10.1016/j.jfma.2015.10.016

[13] Kruisselbrink, R., Kwizera, A., Crowther, M., et al. (2016) Modified Early Warning Score (MEWS) Identifies Critical Illness among Ward Patients in a Resource Restricted Setting in Kampala, Uganda: A Prospective Observational Study. PLoS ONE, 11, e0151408. https://doi.org/10.1177/1062860612450459

[14] Kavanaugh, M.J., So, J.D., Park, P.J., et al. (2017) Validation of the Intensive Care Unit Early Warning Dashboard: Quality Improvement Utilizing a Retrospective Case-Control Evaluation. Telemedicine and e-Health, 23, 88-95.

https://doi.org/10.1089/tmj.2016.0073

[15] Urban, R.W., Mumba, M., Martin, S.D., et al. (2015) Modified Early Warning System as a Predictor for Hospital Admissions and Previous Visits in Emergency Departments. Advanced Emergency Nursing Journal, 37, 281-289. https://doi.org/10.1097/TME.0000000000000076

[16] NannanPanday, R.S., Minderhoud, T.C., Alam, N., et al. (2017) Prognostic Value of Early Warning Scores in the Emergency Department (ED) and Acute Medical Unit (AMU): A Narrative Review. European Journal of Internal Medicine, 45, 20-31. https://doi.org/10.1016/j.ejim.2017.09.027

[17] Nishijima, I., Oyadomari, S., Maedomari, S., et al. (2016) Use of a Modified Early Warning Score System to Reduce the Rate of In-Hospital Cardiac Arrest. Journal of Intensive Care, 4, 12. https://doi.org/10.1186/s40560-016-0134-7

[18] Wang, L., Du, J., Chen, W., et al. (2015) Application Analysis of the Improved Early Warning Scoring System in the Nursing of "Potential Critical Patients" in Cardiothoracic Surgery. Chinese General Medicine, No. 14, 1680-1683.

[19] Yang, L., Zhang, Y., Chen, F., et al. (2016) Application of Modified Early Warning Score in Disease Risk Management of Elderly Inpatients in Digestive Surgery Department. Nursing Research, No. 13, 1633-1635.

[20] Mei, L., Chen, W. and Li, C. (2016) Study on the Evaluation Value of Improved Early Warning Score for the Severity and Prognosis of In-Patients with Coronary Heart Disease. Journal of Nursing, 23, 50-53.

[21] Li, Y., Ye, H., Tang, M., et al. (2011) Study on the Prognostic Value of Improved Early Warning Score in Patients with Severe Pancreatitis. Journal of Clinical Rational Drug Use, No. 2, 27-28.

[22] Zhao, T. and Qin, L. (2018) The Evaluation Value of MEWS Score on Admission for Prognosis of Patients in Emergency Intensive Care Unit. Henan Medical Research, No. 2, 205-208.

[23] Su, Y., Huang, L. and Yu, L. (2016) Application Analysis of the Improved Early Warning Scoring System in the Nursing of "Potential Critical Patients" in Cardiothoracic Surgery. Contemporary Nurse, No. 4, 57-59.

[24] Sandroni, C., Nolan, J., Cavallaro, F., et al. (2007) In-Hospital Cardiac Arrest: Incidence, Prognosis and Possible Measures to Improve Survival. Intensive Care Medicine, 33, 237-245. https://doi.org/10.1007/s00134-006-0326-Z

[25] Delgado-Hurtado, J.J., Berger, A. and Bansal, A.B. (2016) Emergency Department Modified Early Warning Score Association with Admission, Admission Disposition, 
Mortality, and Length of Stay. Journal of Community Hospital Internal Medicine Perspectives, 6, 31456. https://doi.org/10.3402/jchimp.v6.31456

[26] Ye, J., Zhao, Y., Ju, J., et al. (2017) Building and Verifying a Severity Prediction Model of Acute Pancreatitis (AP) Based on BISAP, MEWS and Routine Test Indexes. Clinics and Research in Hepatology and Gastroenterology, 41, 585-591. https://doi.org/10.1016/j.clinre.2016.11.013

[27] Skaletzky, S.M., Raszynski, A. and Totapally, B.R. (2011) Validation of a Modified Pediatric Early Warning System Score. Clinical Pediatrics, 51, 431-435. https://doi.org/10.1177/0009922811430342

[28] Ludikhuize, J., Smorenburg, S.M., de Rooij, S.E., et al. (2012) Identification of Deteriorating Patients on General Wards; Measurement of Vital Parameters and Potential Effectiveness of the Modified Early Warning Score. Journal of Critical Care, 27, 424-427. https://doi.org/10.1016/j.jcrc.2012.01.003

[29] Subbe, C.P., et al. (2001) Validation of a Modified Early Warning Score in Medical Admissions. QJM, 94, 521-526.

[30] Tirotta, D., Gambacorta, M., La Regina, M., et al. (2017) Evaluation of the Threshold Value for the Modified Early Warning Score (MEWS) in Medical Septic Patients: A Secondary Analysis of an Italian Multicentric Prospective Cohort (SNOOPII Study). QJM: An International Journal of Medicine, 110, 369-373. https://doi.org/10.1093/qjmed/hcw229

[31] Gerry, S., Birks, J., Bonnici, T., et al. (2017) Early Warning Scores for Detecting Deterioration in Adult Hospital Patients: A Systematic Review Protocol. BMJ Open, 7, e019268. https://doi.org/10.1136/bmjopen-2017-019268

[32] Green, M., Lander, H., Snyder, A., et al. (2018) Comparison of the between the Flags Calling Criteria to the MEWS, NEWS and the Electronic Cardiac Arrest Risk Triage (eCART) Score for the Identification of Deteriorating ward Patients. Resuscitation, 123, 86-91. https://doi.org/10.1016/j.resuscitation.2017.10.028

[33] Lu, X., Yang, Z. and Gu, Y. (2014) Application of Early Warning Score in Identifying "Potential Critical Illness" in Surgical Wards. Nursing Practice and Research, No. 11, 58-59.

[34] Huang, W., Cui, M., Jiang, Y., et al. (2016) UK National Early Warning Scale and Its Clinical Application Research Progress. Journal of Nursing, No. 6, 101-104.

[35] Wuytack, F., Meskell, P., Conway, A., et al. (2017) The Effectiveness of Physiologically Based Early Warning or Track and Trigger Systems after Triage in Adult Patients Presenting to Emergency Departments: A Systematic Review. BMC Emergency Medicine, 17, 38. https://doi.org/10.1186/s12873-017-0148-Z

[36] Alam, N., Hobbelink, E.L., van Tienhoven, A.J., et al. (2014) The Impact of the Use of the Early Warning Score (EWS) on Patient Outcomes: A Systematic Review. Resuscitation, 85, 587-594. https://doi.org/10.1016/j.resuscitation.2014.01.013 